\title{
Effect of VX-765 on the transcriptome profile of mice spinal cords with acute injury
}

\author{
JING CHEN $^{1-3^{*}}$, YU-QING CHEN ${ }^{1-3 *}$, SAI-NAN WANG ${ }^{1-3}$, FEI-XIANG DUAN $^{1,2}$, \\ YU-JIAO SHI ${ }^{1,2}$, SHU-QIN DING ${ }^{1,2}$, JIAN-GUO HU ${ }^{1,2}$ and HE-ZUO LÜ $\ddot{H}^{1-3}$ \\ ${ }^{1}$ Clinical Laboratory; ${ }^{2}$ Anhui Key Laboratory of Tissue Transplantation, The First Affiliated Hospital of Bengbu \\ Medical College, Bengbu, Anhui 233004; ${ }^{3}$ Department of Immunology, Bengbu Medical College, \\ Bengbu, Anhui 233030, P.R. China
}

Received June 07, 2019; Accepted November 18, 2019

DOI: $10.3892 / \mathrm{mmr} .2020 .11129$

\begin{abstract}
Previous studies have shown that caspase-1 plays an important role in the acute inflammatory response of spinal cord injury (SCI). VX-765, a novel and irreversible caspase-1 inhibitor, has been reported to effectively intervene in inflammation. However, the effect of VX-765 on genome-wide transcription in acutely injured spinal cords remains unknown. Therefore, in the present study, RNA-sequencing (RNA-Seq) was used to analyze the effect of VX-765 on the local expression of gene transcription $8 \mathrm{~h}$ following injury. The differentially expressed genes (DEGs) underwent enrichment analysis of functions and pathways by Gene Ontology and Kyoto Encyclopedia of Genes and Genomes analyses, respectively. Parallel analysis of western blot confirmed that VX-765 can effectively inhibit the expression and activation of caspase-1. RNA-Seq showed that VX-765 treatment resulted in 1,137 upregulated and 1,762 downregulated DEGs. These downregulated DEGs and their associated signaling pathways, such as focal adhesion, cytokine-cytokine receptor interaction, leukocyte transendothelial migration, extracellular matrix-receptor interaction, phosphatidylinositol 3-kinase-protein kinase B, Rap1 and hypoxia inducible factor-1 signaling pathway, are mainly associated with inflammatory response, local hypoxia, macrophage differentiation, adhesion migration and apoptosis of local cells. This suggests that the application of VX-765 in
\end{abstract}

Correspondence to: Professor He-Zuo Lü, Clinical Laboratory, The First Affiliated Hospital of Bengbu Medical College, 287 Chang Huai Road, Bengbu, Anhui 233004, P.R. China

E-mail: 1hz233003@163.com

Professor Jian-Guo Hu, Anhui Key Laboratory of Tissue Transplantation, The First Affiliated Hospital of Bengbu Medical College, 287 Chang Huai Road, Bengbu, Anhui 233004, P.R. China E-mail: jghu9200@163.com

*Contributed equally

Key words: RNA-sequencing, mice, spinal cord injury, VX-765, caspase-1 the acute phase can improve the local microenvironment of SCI by inhibiting caspase-1. However, whether VX-765 can be used as a therapeutic drug for SCI requires further exploration. The sequence data have been deposited into the Sequence Read Archive (https://www.ncbi.nlm.nih.gov/sra/PRJNA548970).

\section{Introduction}

Spinal cord injury (SCI) covers various types of damage to the spinal cord. According to the severity of injury, the symptoms may vary, ranging from pain to complete loss of movement and sensory function. SCI affects millions of people worldwide, usually for life (1). To date, there is no effective treatment. Therefore, finding new treatment methods for patients with SCI is crucial. However, exploring the pathogenesis of SCI and finding effective treatment strategies has been a great challenge for researchers.

Previous studies have suggested that, in injured spinal cords, the inflammasome can activate inflammatory caspases and cytokines of the interleukin (IL)-1 family (IL-1 $\beta$ and IL-18) by identifying host-derived damage-associated molecular patterns (2-4). VX-765, also known as Belnacasan, is an inhibitor of IL-1-converting enzyme (caspase-1), which controls the generation of IL-1 $\beta$ and IL-18 (5-7). VX-765 has been shown to inhibit acute seizures and chronic epilepsy in preclinical models (8). Therefore, using VX-765 to inhibit caspase-1, the common converting enzyme of these two inflammatory factors, in the acute stage of SCI might be an effective anti-inflammatory intervention. However, the exact mechanism is not entirely clear. The aim of the present study was to use VX-765 $8 \mathrm{~h}$ after SCI, in order to analyze the transcription of the local genes, using RNA-sequencing (RNA-Seq). Next, through bioinformatics analysis and reverse transcription-quantitative PCR (RT-qPCR), key molecular and signaling pathways were screened and identified, providing a new theoretical and experimental basis for SCI clinical treatment.

\section{Materials and methods}

Animals. A total of 27 healthy and clean C57BL/6 female mice (weight, 18-20 g; age, 8 weeks old; Chang Zhou Cavens Laboratory Animal Ltd.) were used in this study (Fig. 1). 
Animal care following surgery complied with the regulations for the management of experimental animals (revised by the Ministry of Science and Technology of China in June 2004). The study was approved by the Institutional Committee on Animal Care, Use and Research of the Bengbu Medical College (approval no. 2017037).

Contusive SCI and drug injection. An Infinite Horizon impactor (Precision Systems \& Instrumentation) was used to perform contusive SCI, as previously described (9-11). The mice were first anesthetized with pentobarbital sodium $(50 \mathrm{mg} / \mathrm{kg}$, intraperitoneally) and then the T9 lamina was excised. The spine was stabilized by clamping the T7 and T11 spinous processes, and then a moderate SCI model was created using a rod $(1.3 \mathrm{~mm}$ in diameter) with a force of 50 Kdynes and a dwell time of 0 sec. Sham-operated (sham) mice only received a laminectomy without contusive injury.

The spinal cord-injured mice were randomly assigned to the DMSO control or VX-765 injection groups (9 mice in every group). Mice were intraperitoneally injected with DMSO or VX-765 (100 mg/kg prepared in DMSO) immediately following injury. Since the aim was to investigate the effect of VX-765 on local gene transcription in the acute stage of SCI, all specimens were collected $8 \mathrm{~h}$ after SCI.

Identification of the effect of VX-765 on caspase-1 and 3 activities in injured spinal cords by western blot analysis. A total of $8 \mathrm{~h}$ following surgery, the mice were euthanized referring to previous references $(12,13)$ with an overdose of pentobarbital sodium $(80 \mathrm{mg} / \mathrm{kg}$, intraperitoneally) and perfused with $10 \mathrm{ml}$ PBS. An overdose of pentobarbital sodium can make the mice lose consciousness within 2 min with minimal pain and distress. Subsequent PBS perfusion can make animals die (no spontaneous breathing and blink reflex) within 2-3 min with no pain and distress, while spinal cord samples were taken $(0.5 \mathrm{~cm}$, including the injury center, $\mathrm{n}=6$ in every group). Total protein was extracted from the spinal cords using a mammalian protein extraction kit (cat. no. C600589; Sangon Biotech Co., Ltd.) and western blot analysis was performed as previously described (14). For the caspase-3 analysis, the primary antibodies used were rabbit anti- $\beta$-actin (1:2,000; cat no. BL005B; Biosharp) and rabbit anti-caspase- 3 antibody (1:1,000; cat no. ab13847; Abcam). The secondary antibody used was horseradish peroxidase (HRP)-conjugated goat anti-rabbit IgG (1:10,000; cat. no. BL003A; Biosharp). As the molecular weights of $\beta$-actin and pro-caspase-1 are similar (15), when western blot analysis was performed, rabbit monoclonal anti-caspase-1+p10+p12 antibody (1:1,000; cat. no. ab179515; Abcam) was first used to incubate for $12 \mathrm{~h}$ at $4^{\circ} \mathrm{C}$ and detect protein levels, then the restore western blot stripping buffer (cat. no. 21059; Thermo Fisher Scientific, Inc.) was used to wash off the antibody on the membrane. For the stripping, the blot was placed in $20 \mathrm{ml}$ restore western blot stripping buffer and incubated for $15 \mathrm{~min}$ at $37^{\circ} \mathrm{C}$. Then, the blot was removed and washed 5 min with PBS three times. To test for complete removal of the HRP labeled secondary antibody, the membrane was incubated with a SuperSignal West Working solution (cat. no. 34095; Thermo Fisher Scientific, Inc.) for $5 \mathrm{~min}$ at room temperature and exposed to film. If no signal is detected using a 5-min exposure, the HRP-conjugated secondary antibody

\section{Illustration of the experimental design}

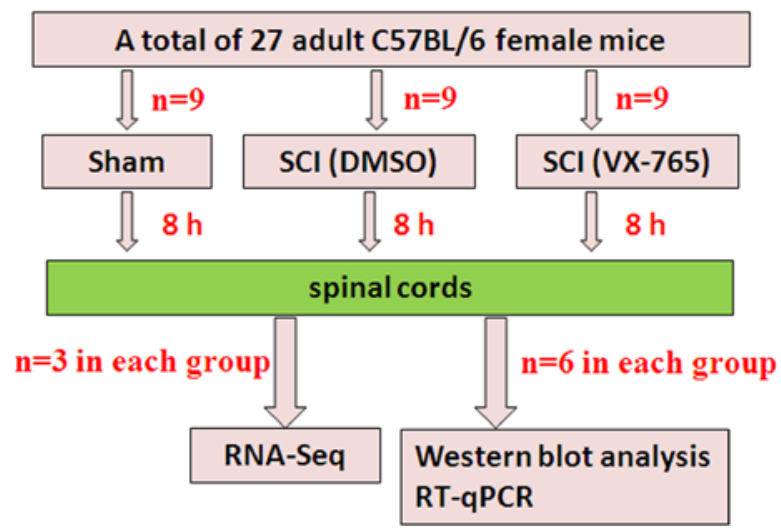

Figure 1. Illustration of the experimental design. SCI, spinal cord injury; RT-qPCR, reverse transcription-quantitative PCR; RNA-Seq, RNA-sequencing.

had been successfully removed. To test for complete removal of the primary antibody, the membrane was incubated with the HRP-labeled secondary antibody, followed by washing $5 \mathrm{~min}$ in $1 \mathrm{X}$ TBST buffer containing $0.01 \mathrm{M}$ Tris, $0.15 \mathrm{M} \mathrm{NaCl}$ and 0.1\% Tween-20 (cat. no. C520009; Sangon Biotech Co., Ltd.) three times. Then, membrane was incubated in SuperSignal West Working Solution for $5 \mathrm{~min}$ at room temperature and exposed to film again. If no signal is detected with a 5-min exposure, the primary antibody has been successfully removed from the antigen. After determining that the membrane was properly stripped, the second immunoprobing experiment of anti- $\beta$-actin antibody was performed as previously described (14).

RNA isolation, quantification and qualification. A total of $8 \mathrm{~h}$ following surgery, mice were euthanized with an overdose of pentobarbital sodium $(80 \mathrm{mg} / \mathrm{kg}$, intraperitoneally) $(12,13)$ and perfused with $10 \mathrm{ml} \mathrm{PBS}$, and the spinal cords $(0.5 \mathrm{~cm}$, including the injury center) were removed. Total RNA was extracted from spinal cords and purified, as previously described (16).

Library preparation and transcriptome sequencing. The sequencing libraries were produced using NEBNext ${ }^{\circledR}$ Ultra $^{\mathrm{TM}}$ RNA Library Prep kit for Illumina ${ }^{\circledR}$ (New England Biolabs) as previously described (13). Finally, the 125-bp/150-bp paired-end reads were obtained and sequenced on an Illumina Hiseq platform. The sequence data have been deposited into Sequence Read Archive (https://www.ncbi.nlm.nih.gov/sra/ PRJNA548970).

Differentially expressed gene (DEG) analysis. Prior to DEG analysis, the gene expression statistics were analyzed using RSEM software (v1.3.1; http://deweylab.biostat.wisc.edu/ $\mathrm{rsem} /$ ) to convert the read count numbers to Fragments Per Kilobase of transcript per Million fragments mapped (FPKM) and Principal Component Analysis (PCA) was made to determine the similarities and differences in the data. Differential gene expression in the three groups was analyzed as previously described (16), using the DESeq software (http://www. 
Table I. PCR primers used in the study.

\begin{tabular}{llll}
\hline Gene & GenBank Accession no. & \multicolumn{1}{c}{ Forward primer 5' $\rightarrow 3^{\prime}$} & Reverse primer 5' $\rightarrow 3^{\prime}$ \\
\hline Caspase-1 & NM_009807.2 & CGTACACGTCTTGCCCTCAT & GGGCAGGCAGCAAATTCTTT \\
IL-1 & NM_008361.4 & ACAACTGCACTACAGGCTCC & TGGGTGTGCCGTCTTTCATT \\
LCN2 & NM_008491.1 & ACAACCAGTTCGCCATGGTAT & AAGCGGGTGAAACGTTCCTT \\
Nlrp3 & NM_145827 & GACCGTGAGGAAAGGACCAG & GGCCAAAGAGGAATCGGACA \\
Pecam1 & XM_021175967.1 & GTACCAATCCAGGTGTGCGA & TTTTCGGACTGGCAGCTGAT \\
CD34 & NM_133654.3 & ACCACAGACTTCCCCAACTG & CATATGGCTCGGTGGGTGAT \\
Gbp7 & BK005760 & GGACGTGTCATCACAGCAGA & CCAACTGGTCCTCTGGCATT \\
Icos1 & NM_015790.3 & GAACCCACAGGAAACCCACA & GTATAGCTTCGGTGGGGACG \\
Hpdg & XM_021170107.1 & CTTCGAAGCACGGCATCATC & TGGCAATGGTTGATGGGTGTA \\
Cc19 & NM_011338.2 & CAGGCCGGGCATCATCTTTA & TGGCAGTTCACACCCTTCTC \\
Rel & NM_009044.2 & TACTCGGCCTCTGAGTGTGA & GGCCTAGCCTGGCATTACAT
\end{tabular}

IL, interleukin; Lcn2, lipocalin-2; Nlrp3, nucleotide-binding oligomerization domain, leucine-rich repeat and pyrin domain-containing 3; Pecam1, platelet endothelial cell adhesion molecule; Gbp7, guanylate binding protein 7; Icos1, inducible T-cell COStimulator; Hpdg, 15-hydroxyprostaglandin dehydrogenase; Ccl9 chemokine (C-C motif) ligand 9.

A
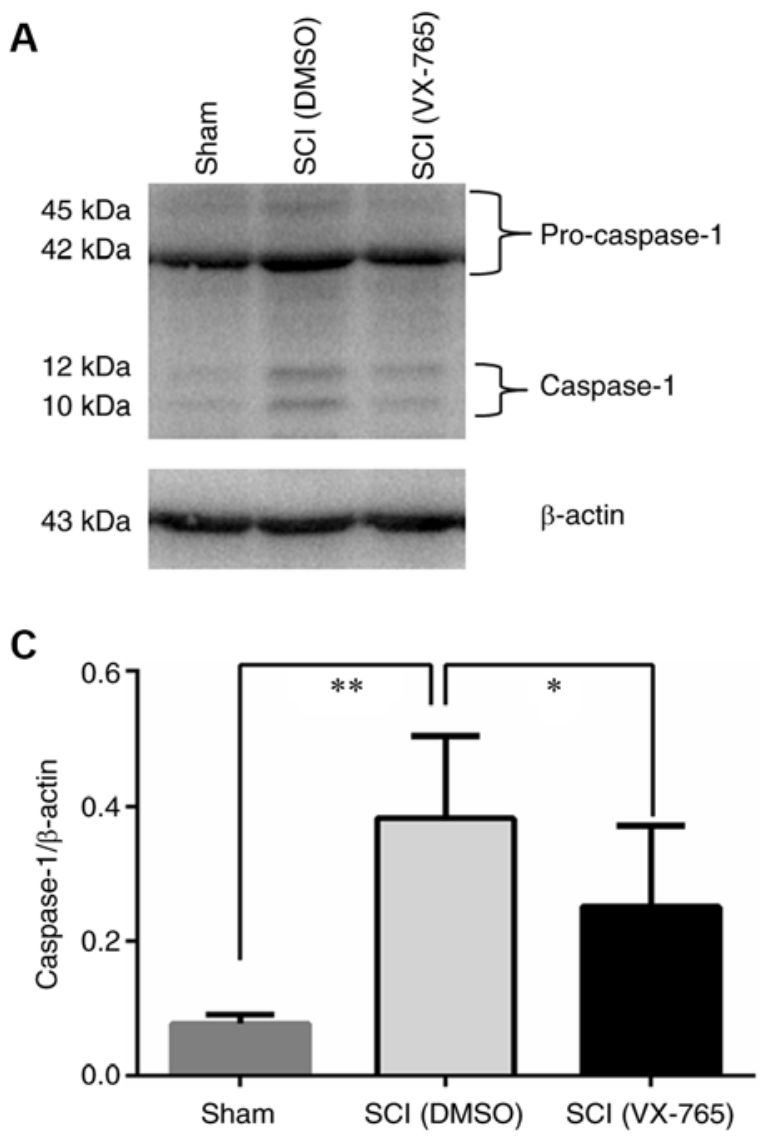

B

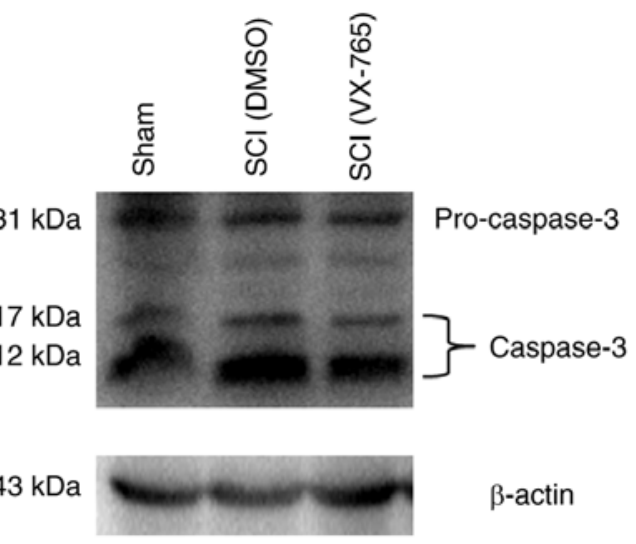

D

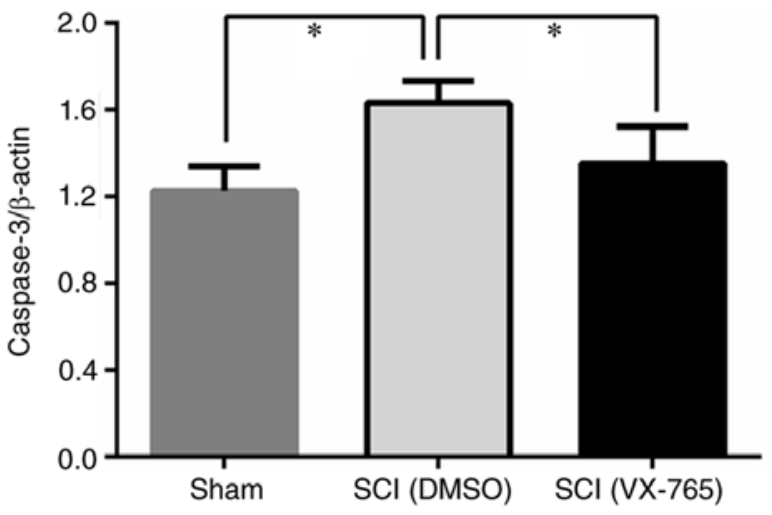

Figure 2. Western blot analysis the effect of VX-765 on caspase-1 activity in injured spinal cord. (A) Representative western blotting results of the expression of pro-caspase-1 (42 and $45 \mathrm{kDa}$ ), caspase-1 (10 and $12 \mathrm{kDa}$ ) and $\beta$-actin (43 kDa). (B) Representative western blot results of the expression of pro-caspase-3 (34 kDa), caspase-3 (17 and $12 \mathrm{kDa})$ and $\beta$-actin (43 kDa). The statistical graphs of the expression of (C) caspase-1 and (D) caspase-3 in sham, injured spinal cords in DMSO control and VX-765 treatment groups. Data represent the mean \pm standard deviation $(\mathrm{n}=6)$. ${ }^{*} \mathrm{P}<0.05$ and ${ }^{* *} \mathrm{P}<0.01($ analysis of variance). $\mathrm{SCI}$, spinal cord injury.

bioconductor.org/). Benjamini and Hochberg's approach was used to control the false discovery rate and adjust the P-values. An adjusted $\mathrm{P}<0.05$ was defined as a standard for significant differences in gene expression.
Gene ontology (GO) and kyoto encyclopedia of genes and genomes (KEGG) enrichment analysis of DEGs. GO and KEGG analysis was performed using GOseq $\mathrm{R}$ package and KOBAS software, as previously described (16). 
Table II. Summary of sequence assembly after Illumina sequencing.

\begin{tabular}{lccccccc}
\hline Sample name & Raw reads & Clean reads & Clean bases & Error rate $(\%)$ & Q20 (\%) & Q30 (\%) & GC content (\%) \\
\hline Sham1 & 56509230 & 55796658 & $8.37 \mathrm{G}$ & 0.03 & 97.73 & 93.95 & 51.23 \\
Sham2 & 48848744 & 48226002 & $7.23 \mathrm{G}$ & 0.03 & 97.60 & 93.67 & 51.71 \\
Sham3 & 58228350 & 57459748 & $8.62 \mathrm{G}$ & 0.03 & 97.67 & 93.78 & 51.42 \\
SCI_C1 & 56857568 & 56047834 & $8.41 \mathrm{G}$ & 0.03 & 97.80 & 94.08 & 50.96 \\
SCI_C2 & 52518750 & 51674904 & $7.75 \mathrm{G}$ & 0.03 & 97.80 & 94.11 & 50.99 \\
SCI_C3 & 62676868 & 61993808 & $9.30 \mathrm{G}$ & 0.03 & 97.80 & 94.09 & 51.09 \\
SCI_V1 & 48440020 & 47736336 & $7.16 \mathrm{G}$ & 0.02 & 97.92 & 94.39 & 51.23 \\
SCI_V2 & 55927984 & 54923592 & $8.24 \mathrm{G}$ & 0.03 & 97.86 & 94.31 & 51.33 \\
SCI_V3 & 59647724 & 59047622 & $8.86 \mathrm{G}$ & 0.03 & 97.90 & 94.30 & 51.09
\end{tabular}

Sham: Sham1, Sham2, Sham3; SCI (DMSO): SCI_C1, SCI_C2, SCI_C3; SCI (VX-765): SCI_V1, SCI_V2, SCI_V3; Q20: The percentage of bases with a Phred value $>20$; Q30: The percentage of bases with a Phred value $>30$. SCI, spinal cord injury.

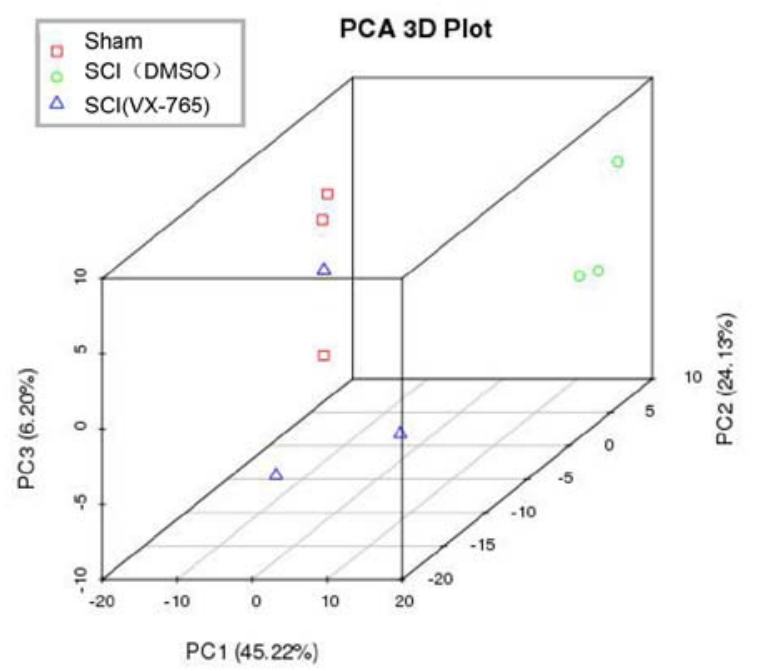

Figure 3. PCA analysis. PCA analysis was performed using three principal components (PC1, 2 and 3 ) to demonstrate the source of variance $(\mathrm{n}=3)$. PCA, principal component analysis; SCI, spinal cord injury.

$R T$ - $q P C R$. To validate RNA-Seq results, nine DEGs were randomly selected and verified by RT-qPCR, as previously described (16). PCR primer sequences are listed in Table I. The relative quantitative results of each group $(n=6)$ of genes were calculated according to the $2^{-\Delta \Delta \mathrm{Cq}}$ formula (17).

Statistical analysis. Statistical values of western blot analysis and RT-qPCR ( $=6 /$ group) were presented as mean \pm standard deviation. The data were analyzed using one-way analysis of variance followed by Student-Newman-Keuls tests. $\mathrm{P}<0.05$ was considered to indicate a statistically significant difference. All tests were performed using SPSS 16.0 (SPSS, Inc.).

\section{Results}

Effect of VX-765 on caspase-1 activity in injured spinal cords. To verify the effect of VX-765 on caspase-1 activity in injured spinal cords, the homogenate extracts obtained from
DMSO and VX-765-treated spinal cords were detected by western blot analysis. As the molecular weights of $\beta$-actin and pro-caspase-1 are similar (14), when western blot analysis was carried out, anti-caspase-1+p10+p12 antibody was first used to incubate and detect protein levels, and then stripping buffer was used to wash off the antibody on the membrane. Then, anti- $\beta$-actin antibody incubation and detection was carried out. As shown in Fig. 2A and B, no significant differences were observed in the 43 and $45 \mathrm{kDa}$ pro-caspase- 1 bands and $31 \mathrm{kDa}$ pro-caspase-3 band. However, the 10 and $12 \mathrm{kDa}$ caspase-1 bands (Fig. 2A) and the 17 and $12 \mathrm{kDa}$ caspase-3 bands (Fig. 2B) in the SCI (DMSO) group were significantly increased compared with those in the sham group $(\mathrm{P}<0.05)$. In VX-765 group, these bands (Fig. 2A and B) were significantly decreased compared with those in the DMSO control group $(\mathrm{P}<0.05)$. Fig. $2 \mathrm{C}$ and $\mathrm{D}$ show a significant difference among the three groups $(n=6, P<0.01$ or 0.05$)$. These results showed that SCI can induce the activities of caspase-1 and 3, and VX-765 can inhibit their activities in injured spinal cords.

Identification of expressed transcripts in the mouse spinal cords. For the quality assessment of sequencing data, nine cDNA libraries were established, including Sham (Sham1, Sham2 and Sham3), SCI (DMSO; SCI_C1, SCI_C2 and SCI_C3) and SCI (VX-765; SCI_V1, SCI_V2 and SCI_V3). RNA-Seq produced 48,440,020-62,676,868 raw reads for each sample. After filtering out the low-quality reads, the clean reads were from 47,736,336-61,993,808 (97.6-97.92\%; Table II).

In order to identify the source of variation in the original data, PCA analysis was conducted. As shown in Fig. 3, PC1, PC2 and PC3 were 45.22, 24.13 and 6.20\%, respectively, demonstrating that the data could be used for the next analysis.

Effect of VX-765 treatment on gene expression. FPKM and DESeq were used to analyze the gene expression level and differential expression profiles, respectively. The results showed that compared with the sham group there were 4,476 DEGs in the SCI (DMSO) group, including 2,899 up- and 1,577 downregulated genes (Fig. 4A and Table SI). As compared with the SCI (DMSO) group, there were 2,899 DEGs in the 


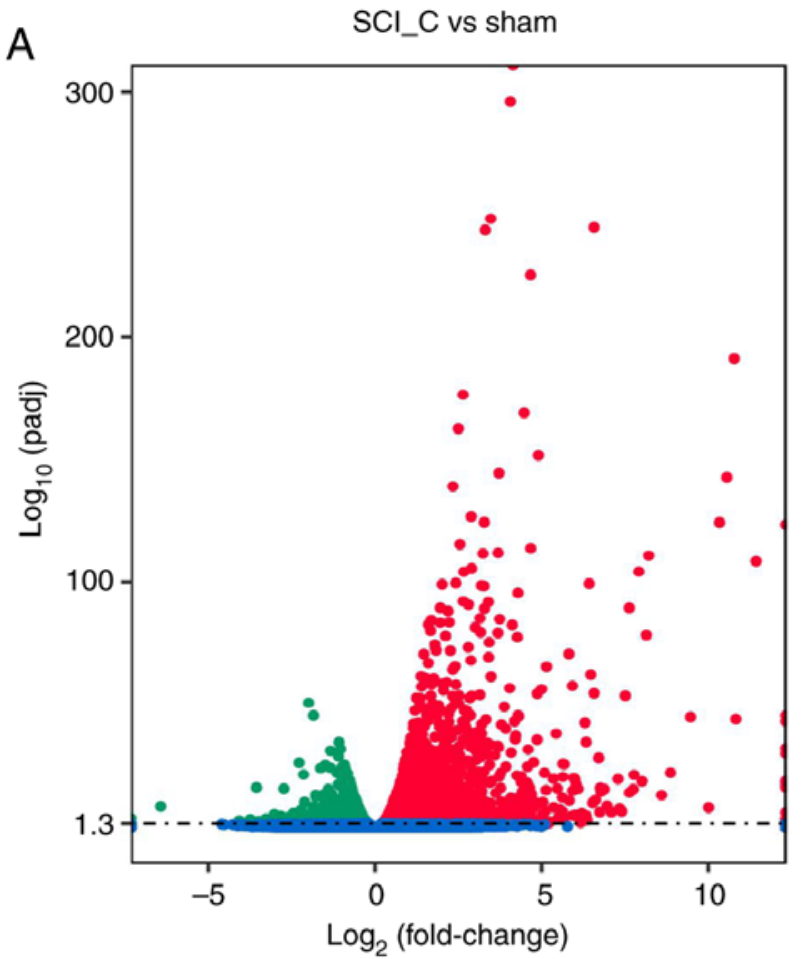

Differential expressed genes (4476)

- Up regulated: 2899

- Down regulated: 1577

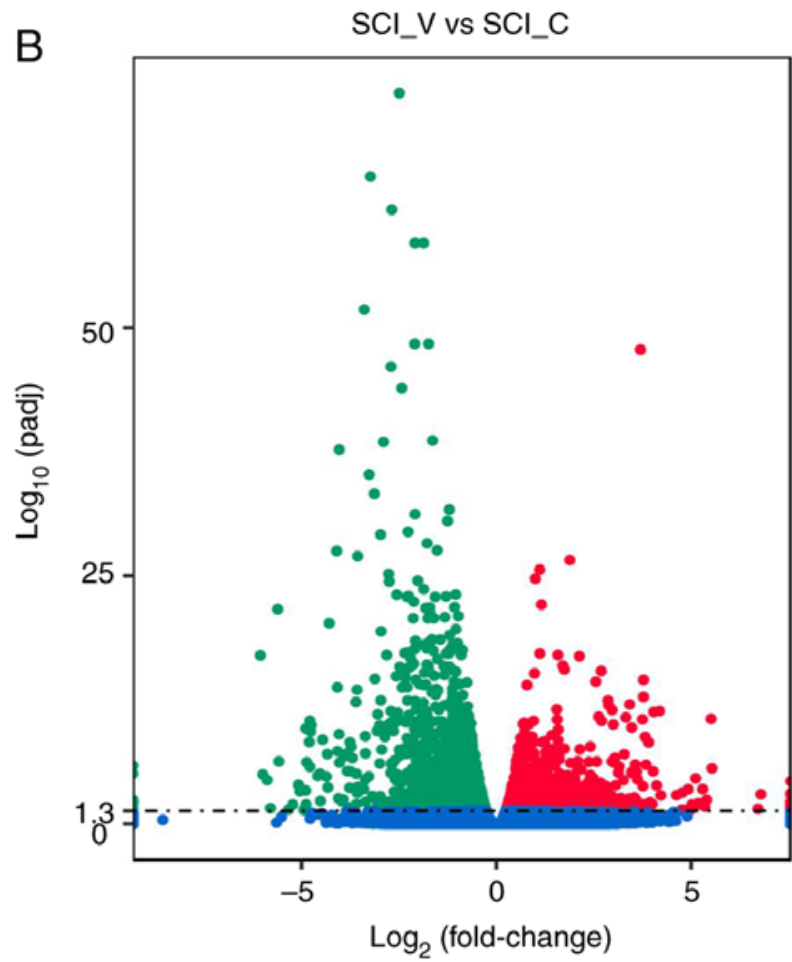

Differential expressed genes (2899)

- Upregulated: 1137

- Downregulated: 1762

Figure 4. Volcano map of DEGs. Red, green and blue dots represent significantly upregulated, downregulated and no changed gene expressions, respectively. (A) SCI (DMSO) vs. Sham; (B) SCI (VX-765) vs. SCI (DMSO). DEGs, differentially expressed genes; SCI, spinal cord injury; IL, interleukin; Seq, sequencing; $\mathrm{CD}$, cluster of differentiation.

SCI (VX-765) group, 1,137 of which were up- and 1,762 were downregulated (Fig. 4B and Table SI).

$R T-q P C R$ identification of DEGs. In order to verify the RNA-Seq results, 11 DEGs were randomly selected from the SCI (VX-765) group, as compared with the SCI (DMSO) group, namely caspase-1,IL-1 $\beta$, Lipocalin-2 (Lcn2), nucleotide-binding oligomerization domain, leucine-rich repeat and pyrin domain-containing 3 (Nlrp3), platelet endothelial cell adhesion molecule (Pecam1), CD34, guanylate binding protein 7 (Gbp7), inducible T-cell COStimulator (Icos1), 15-hydroxyprostaglandin dehydrogenase (Hpdg), chemokine (C-C motif) ligand 9 (Ccl9) and Rel. The RNA-Seq and RT-qPCR results indicated that the expression patterns of these DEGs were similar (Fig. 5).

DEG cluster analysis. The DEGs from different groups were analyzed using FPKM hierarchical cluster analysis. As shown in Fig. 6, DEGs were classified into different expression clusters by hierarchical clustering. These clusters contained up- or downregulated DEGs. Most upregulated DEGs in the SCI (DMSO) group compared with the sham group were in the middle cluster, while downregulated genes were observed in the upper and lower clusters. Compared with the sham group, most upregulated DEGs in the SCI (VX-765) group were in the middle and upper-lower clusters, while downregulated genes were mainly observed in the lower cluster. Compared with the SCI (DMSO) group, some upregulated DEGs in the
SCI (VX-765) group were observed in the upper cluster, while downregulated DEGs were observed in the lower cluster; there were also clusters with no significant differences at the bottom.

GO enrichment analysis of DEGs. Compared with the sham group, $91 \mathrm{GO}$ terms in upregulated DEGs (Fig. 7A, Table SII) and $18 \mathrm{GO}$ terms in downregulated DEGs (Fig. 7B, Table SIII) were found in the SCI (DMSO) group. In the SCI (VX-765) group, one GO term in upregulated DEGs (Fig. 7C, Table SII) and ten GO terms in downregulated DEGs (Fig. 7D, Table SIII) were found, compared with the SCI (DMSO) group. In the SCI (DMSO) group, the downregulated DEGs were most enriched in protein binding, extracellular-glutamate-gated ion channel activity, transmembrane transporter activity, compared with the sham group. The upregulated DEGs were most enriched in anion binding, ribonucleotide binding, purine ribonucleoside triphosphate binding, intracellular signal transduction and chemokine activity. In the SCI (VX-765) group, the downregulated DEGs were most related to binding, signal transduction, transferase, sialyltransferase and $\mathrm{NAD}^{+}$ ADP-ribosyltransferase activity, compared with the SCI (DMSO) group. The upregulated DEGs were only enriched in one term, which was binding.

KEGG enrichment analysis of DEGs. Scatter plots were used to express the KEGG enrichment analysis results for the DEGs. As compared with the sham group, the upregulated DEGs in the 

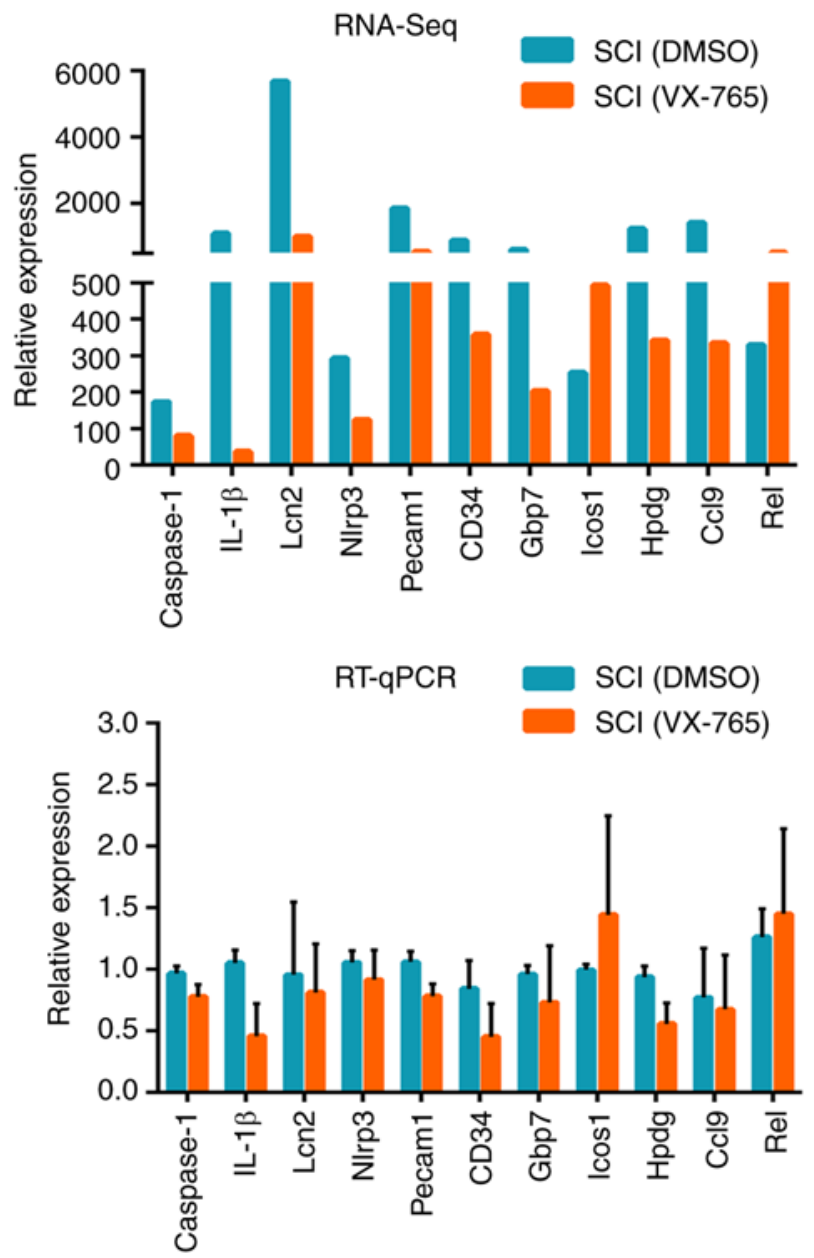

Figure 5. RT-qPCR verification of differentially expressed genes characterized by RNA-Seq. The longitudinal coordinates in RNA-Seq were the mRNA expression level calculated using Transcripts Per Kilobase Million, which can be used directly to compare the difference in gene expression between the samples. The longitudinal coordinates in RT-qPCR were the mRNA expression level calculated using the ${ }^{\Delta \Delta} \mathrm{Cq}$ method and expressed relative to the value in the sham group (designated as 1). All data were calculated with mean \pm standard deviation ( $\mathrm{n}=6$ ). RT-q, reverse transcription-quantitative; Seq, sequencing.

SCI (DMSO) group were most enriched in focal adhesion, apoptosis, tumor necrosis factor, nuclear factor (NF)-кB, Toll-like receptor, phosphatidylinositol 3-kinase (PI3K) protein kinase B $(\mathrm{Akt})$, NOD-like receptor, mitogen associated protein kinase and p53 signaling pathway (Fig. 8A, Table SIV). The downregulated DEGs were most enriched in glutamatergic synapse, endocytosis, Rap1, Hippo and the Ras signaling pathway (Fig. 8B, Table SV). In the SCI (VX-765) group, no enriched signaling pathways were found in the upregulated DEGs (Fig. 8C, Table SIV), compared with the SCI (DMSO) group. The downregulated DEGs were most enriched in focal adhesion, cytokine-cytokine receptor interaction, leukocyte transendothelial migration, extracellular matrix (ECM)-receptor interaction, PI3K-Akt, Rapl and hypoxia inducible factor (HIF)-1 signaling pathway (Fig. 8D, Table SV).

\section{Discussion}

It has been proved that the activation of caspase- 1 needs the recruitment of pro-caspase-1 into the inflammasome and the

\section{Cluster analysis of differentially expressed genes}

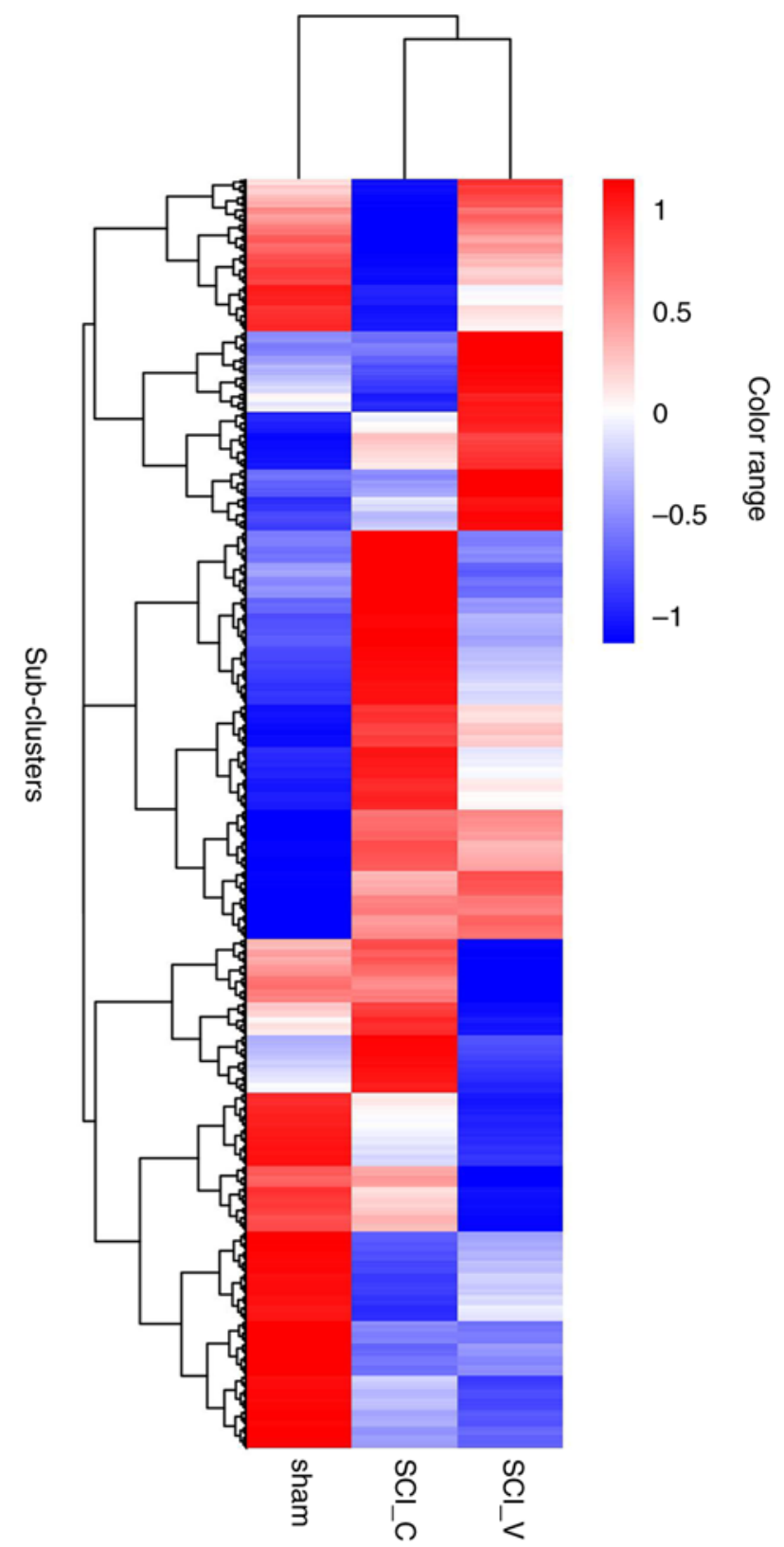

Figure 6. Hierarchical cluster analysis of DEGs. Based on the similarity of gene expression patterns, 8,938 DEGs were divided into multiple expression clusters. The color scheme (blue to red) represents the down to up of the gene expression. sham: Sham group; SCI_C: SCI (DMSO) group; SCI_V: SCI (VX-765) group. SCI, spinal cord injury; GO, gene ontology.

initiation of its self-cleavage. Activated caspase- 1 can further cleave and activate IL-1 family cytokines (such as IL-1 $\beta$ and IL-18), leading to downstream inflammatory cascades $(18,19)$. VX-765 is reported to be a potent and selective inhibitor of caspase-1 (5-7). Although the mechanism is still unclear, this process must be related to inhibiting pro-caspase-1 recruitment to the inflammasome and self-cleavage. Previous studies have shown that the activation of caspase-1 and the resulting expression and activation of IL- $1 \beta$ and IL-18 occur at the site of SCI $(2,20,21)$. Since it is a potent and selective inhibitor, the present study hypothesized that using VX-765 to inhibit caspase-1 in the acute stage of SCI might be an effective anti-inflammatory and anti-apoptotic intervention. To prove 
A

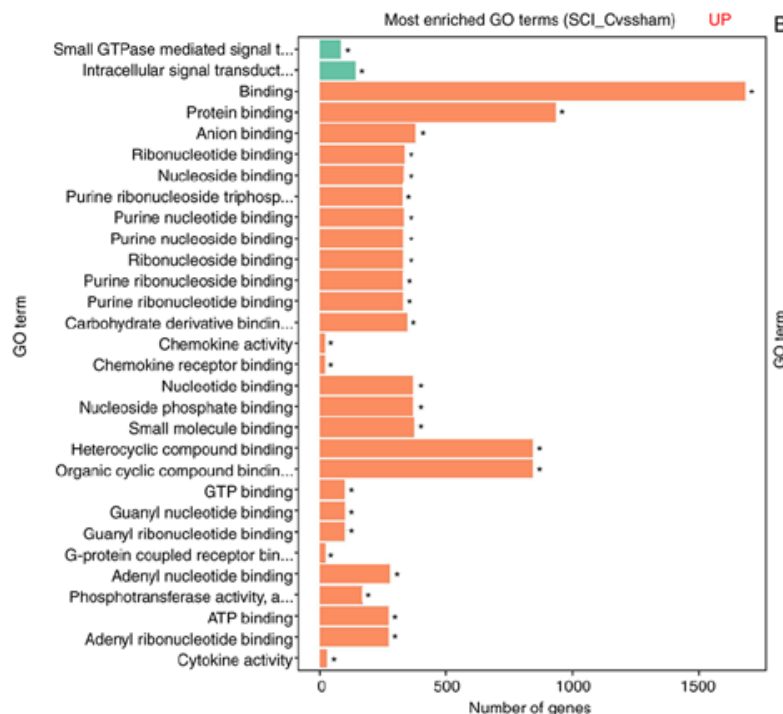

c

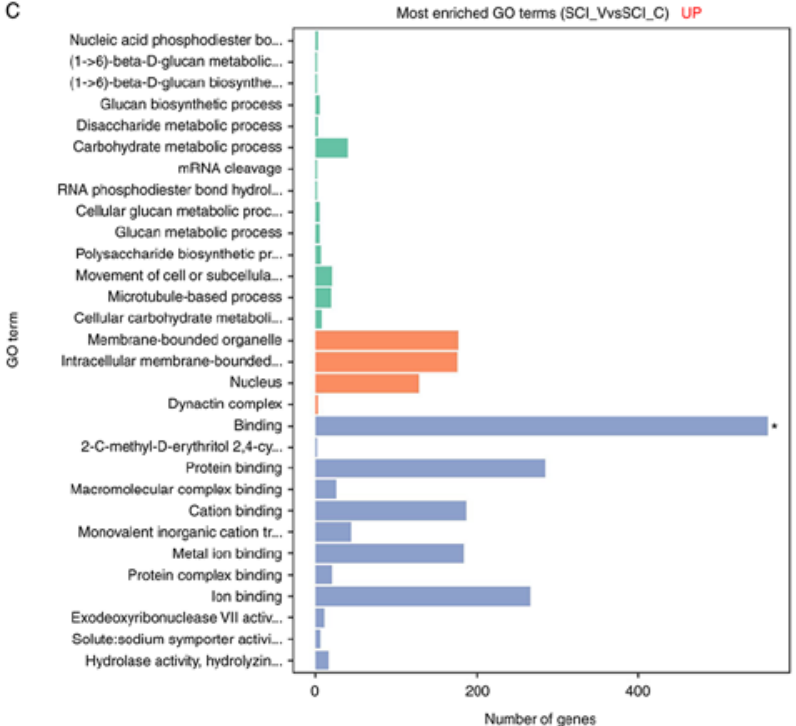

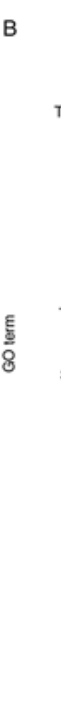

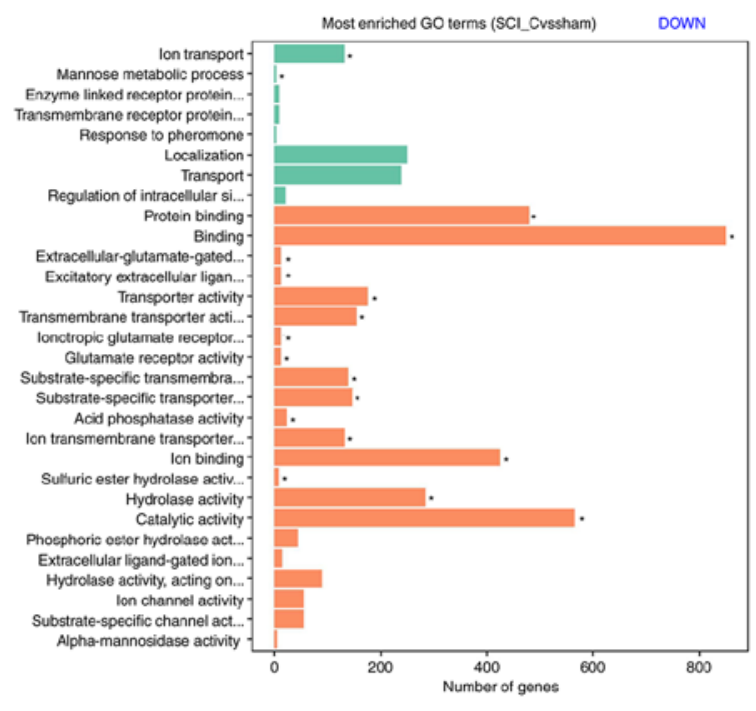

D

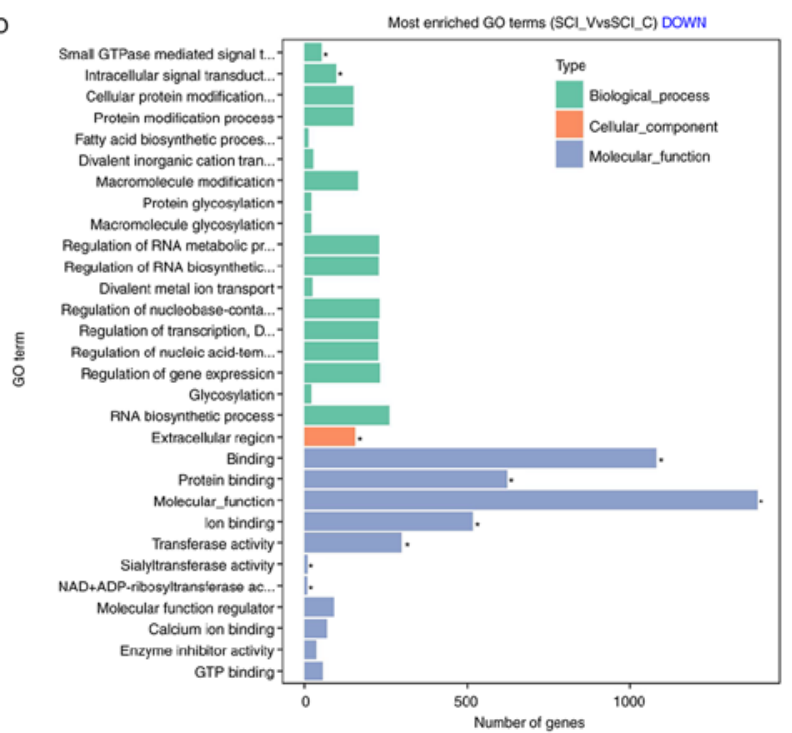

Figure 7. GO enrichment analysis of DEGs. The 30 richest GO terms are listed. (A) Upregulated DEGs in SCI (DMSO) vs. Sham group; (B) downregulated DEGs in SCI (DMSO) vs. Sham group; (C) upregulated DEGs in SCI (VX-765) vs. SCI (DMSO) group; (D) downregulated DEGs in SCI (VX-765) vs. SCI (DMSO) group (D). "P $\leq 0.05$. DEGs, differentially expressed genes; SCI, spinal cord injury; GO, gene ontology.

this hypothesis, RNA-Seq was used in the present study as a detection method to explore the effects of VX-765 on genome-wide transcription in SCI. To identify the effectiveness of VX-765 on caspase-1, western blot analysis was used to detect the pro-caspase-1 and activated caspase-1. The results showed that VX-765 can inhibit caspase-1 activity in injured spinal cords. At the same time, caspase-3, an essential regulator of programmed cell death through apoptosis, was also detected. The results showed that the inhibition of caspase-1 activity can indeed play an anti-apoptotic role. However, these studies are limited and require a high-throughput experiment to confirm these hypotheses. Next, the effects of VX-765 on the local gene transcription of injured spinal cords were characterized by RNA-Seq. Before analyzing the data, the cDNA library quality was examined. $>97 \%$ of the tags were clean and PCA analysis showed that the variation was low. These showed that the cDNA library could be used for the next functional analysis.
The results of RNA-Seq showed that, as compared with the sham group, there were 4,476 DEGs in the SCI (DMSO) group, including 2,899 upregulated and 1,577 downregulated. These were consistent with the present study and other previous reports $(16,22)$, suggesting that the results of this experiment are reliable. As compared with the SCI (DMSO) group, there were 2,899 DEGs in the SCI (VX-765) group, 1,137 of which were upregulated and 1,762 downregulated. To further verify the RNA-seq results, 11 DEGs (caspase-1, IL-1 $\beta$, Lcn2, Nlrp3, Pecam1, CD34, Gbp7, Icos1, Hpdg, Cc19 and Rel) were selected for RT-qPCR. The results showed that the expression patterns of these genes detected by these two methods were similar. These demonstrated that the present RNA-seq results are reliable and can be used for subsequent analysis. These also confirmed that VX-765 can inhibit the expression and activation of caspase-1 and its downstream inflammatory factors. In theory, to be a potent and selective inhibitor of interleukin-converting enzyme/caspase-1, VX-765 can't directly inhibit other types of 
A

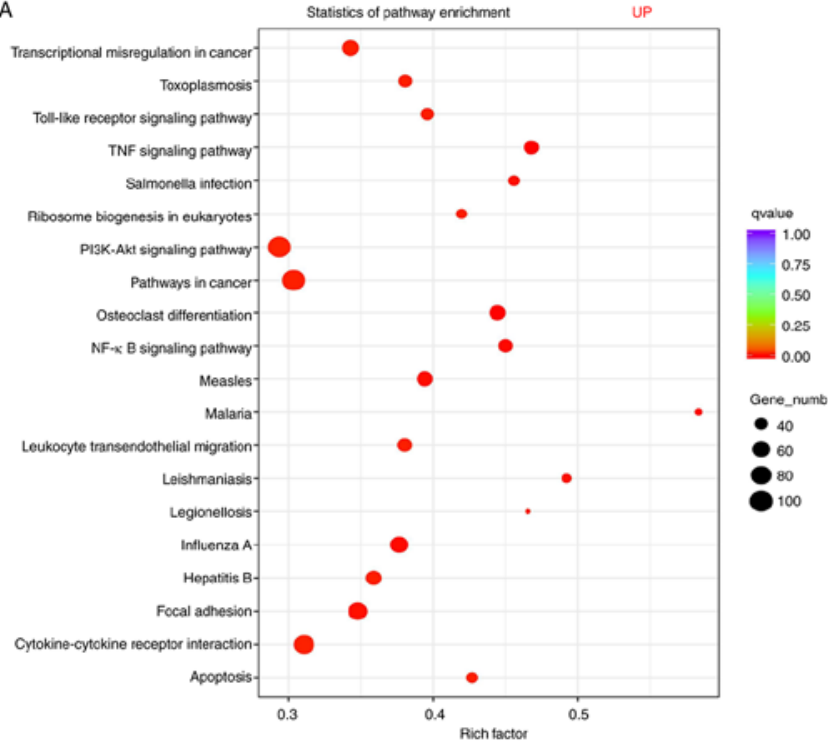

C

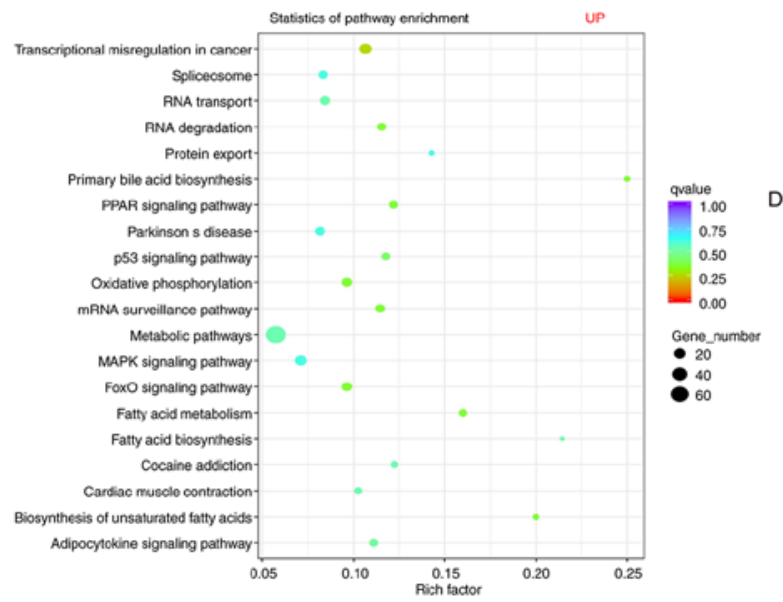

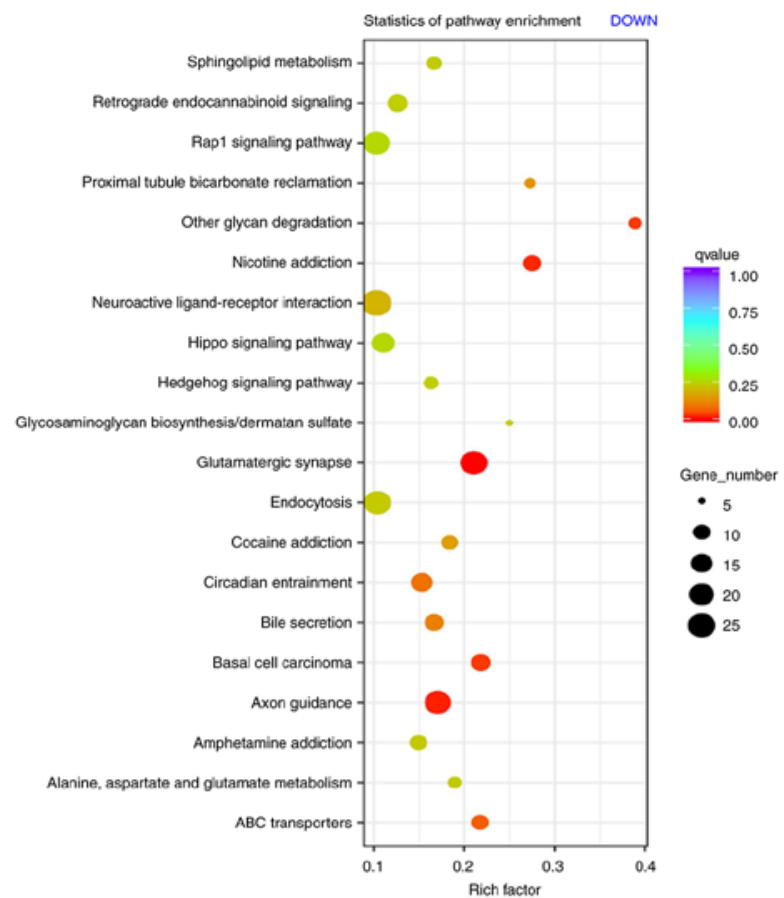

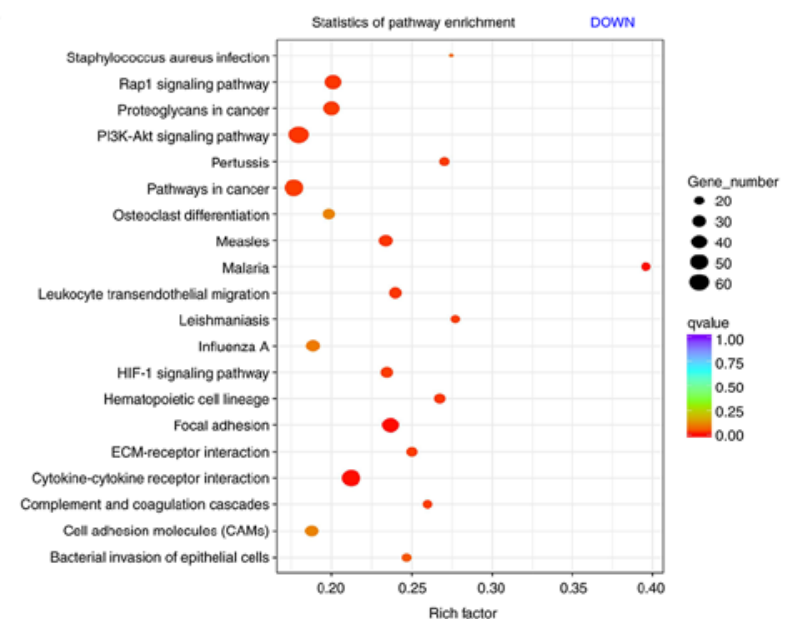

Figure 8. KEGG enrichment analysis of DEGs. The 20 most enriched KEGG pathways were shown. (A) Upregulated DEGs in SCI (DMSO) vs. Sham group; (B) downregulated DEGs in SCI (DMSO) vs. Sham group; (C) upregulated DEGs in SCI (VX-765) vs. SCI (DMSO) group; (D) downregulated DEGs in SCI (VX-765) vs. SCI (DMSO) group (D). DEGs, differentially expressed genes; SCI, spinal cord injury; GO, gene ontology; KEGG, Kyoto, Encyclopedia of Genes and Genomes.

proteins such as matrix metalloproteinases (MMPs). However, in the present RNA-Seq results, 1,762 downregulated genes including MMPs (MMP3, MMP8, MMP11 and MMP16) were found. Although, the possibility of VX-765 inhibiting other types of proteins can't be excluded in this study, it suggests that VX-765 may indirectly inhibit the expression and activity of these proteins by inhibiting caspase-1. This is an interesting topic, which deserves further discussion.

In order to further analyze the DEGs effected by VX-765, GO enrichment analysis was used to determine the distribution of DEGs and enriched cell component, molecular function and biological process GO terms (23). In the SCI (VX-765) group, the downregulated DEGs were most enriched in binding, signal transduction, transferase, sialyltransferase, $\mathrm{NAD}^{+}$ADP-ribosyltransferase activity, compared with the SCI (DMSO) group. The upregulated DEGs were only enriched in one term, which was binding. Next, KEGG was used to analyze signaling pathways associated with treatment of SCI with VX-765. In THE SCI (VX-765) group, no enriched signaling pathways were found in the upregulated DEGs, as compared with the SCI (DMSO) group. The downregulated DEGs were most enriched in focal adhesion, cytokine-cytokine receptor interaction, leukocyte transendothelial migration, ECM-receptor interaction, PI3K-Akt, Rap1 and HIF-1 signaling pathway.

Focal adhesions are specialized intracellular sites in which aggregated integrin receptors interact with extracellular matrices, while extracellular matrices interact with the intracellular actin cytoskeleton $(24,25)$. Focal adhesions are the result of cell-ECM interactions $(24,26)$. The ECM plays an important role in tissue and organ morphogenesis $(27,28)$, as well as the control of cellular activities such as adhesion, 
migration, differentiation, proliferation and apoptosis (29). Cytokines are important regulators and mobilizing factors between cells, which are involved in inflammation, defense, cell growth, differentiation, death and repair processes $(30,31)$. Following VX-765 treatment, the cytokine receptor interaction was inhibited in the sequencing results, involving 56 related genes, such as Csf3, Ifngr1, Ltbr, Tgfbr2 and Il6st. As expected, leukocyte transendothelial migration was also inhibited. During leukocyte division, leukocytes bind to endothelial cell adhesion molecules and then migrate between vascular endothelial cells (32). The migration or infiltration of white blood cells from the blood into tissues is critical to immune surveillance and inflammation (33-35). ECM downregulated in SCI following the injection of VX-765, indicating that VX-765 improves SCI by inhibiting adhesion, migration, differentiation, proliferation and apoptosis through the downregulation of caspase-1 activity.

It has been reported that Akt signaling mediates a variety of extracellular and intracellular signal transduction pathways that regulate macrophage biology, including the production of pro-inflammatory cytokines, phagocytosis, autophagy and homeostasis (36). The PI3K-Akt signaling pathway was downregulated in SCI following the injection of VX-765, indicating that VX-765 can improve SCI by regulating macrophages and inhibiting inflammatory pathways.

The Rap1 signaling pathway plays an important role in regulating cell-cell and cell-matrix interactions by regulating the function of adhesion molecules $(37,38)$. In the present study, the Rapl signaling pathway was enriched in downregulated DEGs of SCI following the injection of VX-765, suggesting that VX-765 may inhibit cell adhesion and polarization by inhibiting the Rap1 signaling pathway, thereby inhibiting inflammation.

HIF- $1 \alpha$ is a specific transcription factor that is activated under hypoxic conditions and its regulated signaling pathway is the backbone of hypoxia signaling $(39,40)$. Immune cells accumulate at the site of inflammation, leaving a rapidly hypoxic environment, which, in turn, causes immune cells to transcribe HIF. In addition, NF- $\kappa$ B positively regulates HIF- $1 \alpha$ expression. The signaling pathway of HIF-1 $\alpha$ and the upstream NF- $\kappa \mathrm{B}$ was inhibited following VX-765 treatment, demonstrating that VX-765 improves the local hypoxic environment of SCI.

In conclusion, the present results demonstrated that VX-765 can lead to gene expression inhibition in acutely injured spinal cords by inhibiting caspase- 1 . These downregulated DEGs and their associated signaling pathways, including focal adhesion, cytokine-cytokine receptor interaction, leukocyte transendothelial migration, ECM-receptor interaction, PI3K-Akt, Rap1 and HIF-1 signaling pathway, are mainly associated with the inflammatory response, local hypoxia, macrophage differentiation, adhesion migration and apoptosis in local cells. This suggests that the application of VX-765 in the acute phase of SCI can improve the local microenvironment of SCI by inhibiting caspase-1. However, whether VX-765 can be used as a therapeutic drug for SCI requires further exploration. Next, detailed research on this subject will be conducted by combining animal models and clinical practice.

\section{Acknowledgements}

Not applicable.

\section{Funding}

The present study was supported by grants from the National Natural Science Foundation of China (grant nos. 81772321 and 81571194).

\section{Availability of data and materials}

All data generated or analyzed during the present study are included in this published article.

\section{Authors' contributions}

HZL and JGH participated in the design of the study. JC, SNW and YQC performed experimental procedures. FXD, YJS and SQD conducted data analysis. All authors read and approved the final manuscript.

\section{Ethics approval and consent to participate}

The present study was approved by the Institutional Committee on Animal Care, Use and Research of the Bengbu Medical College (Approval no. 2017037).

\section{Patient consent for publication}

Not applicable.

\section{Competing interests}

The authors declare that they have no competing interests.

\section{References}

1. Friedli L, Rosenzweig ES, Barraud Q, Schubert M, Dominici N, Awai L, Nielson JL, Musienko P, Nout-Lomas Y, Zhong H, et al: Pronounced species divergence in corticospinal tract reorganization and functional recovery after lateralized spinal cord injury favors primates. Sci Transl Med 7: 302ra134, 2015.

2. de Rivero Vaccari JP, Dietrich WD and Keane RW: Activation and regulation of cellular inflammasomes: Gaps in our knowledge for central nervous system injury. J Cereb Blood Flow Metab 34: 369-375, 2014.

3. Krakauer T: Inflammasomes, autophagy, and cell death: The trinity of innate host defense against intracellular bacteria. Mediators Inflamm 2019: 2471215, 2019.

4. Li L, Tang W and Yi F: Role of inflammasome in chronic kidney disease. Adv Exp Med Biol 1165: 407-421, 2019.

5. Stack JH, Beaumont K, Larsen PD, Straley KS, Henkel GW Randle JC and Hoffman HM: IL-converting enzyme/caspase-1 inhibitor VX-765 blocks the hypersensitive response to an inflammatory stimulus in monocytes from familial cold autoinflammatory syndrome patients. J Immunol 175: 2630-2634, 2005.

6. Zhang Y and Zheng Y: Effects and mechanisms of potent caspase-1 inhibitor VX765 treatment on collagen-induced arthritis in mice. Clin Exp Rheumatol 34: 111-118, 2016.

7. Flores J, Noel A, Foveau B, Lynham J, Lecrux C and LeBlanc AC: Caspase-1 inhibition alleviates cognitive impairment and neuropathology in an Alzheimer's disease mouse model. Nat Commun 9: 3916, 2018.

8. Maroso M, Balosso S, Ravizza T, Iori V, Wright CI, French J and Vezzani A: Interleukin-1 $\beta$ biosynthesis inhibition reduces acute seizures and drug resistant chronic epileptic activity in mice. Neurotherapeutics 8: 304-315, 2011.

9. Wu J, Zhao Z, Kumar A, Lipinski MM, Loane DJ, Stoica BA and Faden AI: Endoplasmic reticulum stress and disrupted neurogenesis in the brain are associated with cognitive impairment and depressive-like behavior after spinal cord injury. J Neurotrauma 33: 1919-1935, 2016. 
10. Horiuchi H, Oshima Y, Ogata T, Morino T, Matsuda S, Miura H and Imamura T: Evaluation of injured axons using two-photon excited fluorescence microscopy after spinal cord contusion injury in YFP-H line mice. Int J Mol Sci 16: 15785-15799, 2015.

11. Galvan MD, LuchettiS, Burgos AM, Nguyen HX, Hooshmand MJ, Hamers FP and Anderson AJ: Deficiency in complement Clq improves histological and functional locomotor outcome after spinal cord injury. J Neurosci 28: 13876-13888, 2008.

12. Liu JQ, Yang D and Folz RJ: A novel bronchial ring bioassay for the evaluation of small airway smooth muscle function in mice. Am J Physiol Lung Cell Mol Physiol 291: L281-L288, 2006.

13. Song XY, Zhou FH, Zhong JH, Wu LL and Zhou XF: Knockout of p75(NTR) impairs re-myelination of injured sciatic nerve in mice. J Neurochem 96: 833-842, 2006.

14. Mehto S, Jena KK, Nath P, Chauhan S, Kolapalli SP, Das SK, Sahoo PK, Jain A, Taylor GA and Chauhan S: The Crohn's disease risk factor IRGM limits NLRP3 inflammasome activation by impeding its assembly and by mediating its selective autophagy. Mol Cell 73: 429-445.e27, 2019.

15. Lin YH, Wu Y, Wang Y, Yao ZF, Tang J, Wang R, Shen L, Ding SQ, Hu JG and Lü HZ: Spatio-temporal expression of Hexokinase-3 in the injured female rat spinal cords. Neurochem Int 113: 23-33, 2018

16. Shi LL, Zhang N, Xie XM, Chen YJ, Wang R, Shen L, Zhou JS, $\mathrm{Hu}$ JG and Lü HZ: Transcriptome profile of rat genes in injured spinal cord at different stages by RNA-sequencing. BMC Genomics 18: 173, 2017.

17. Livak KJ and Schmittgen TD: Analysis of relative gene expression data using real-time quantitative PCR and the 2(-Delta Delta C(T)) method. Methods 25: 402-408, 2001

18. Martinon F, Burns K and Tschopp J: The inflammasome: A molecular platform triggering activation of inflammatory caspases and processing of proIL-beta. Mol Cell 10: 417-426, 2002.

19. Latz E, Xiao TS and Stutz A: Activation and regulation of the inflammasomes. Nat Rev Immunol 13: 397-411, 2013.

20. Mortezaee K, Khanlarkhani N, Beyer C and Zendedel A: Inflammasome: Its role in traumatic brain and spinal cord injury. J Cell Physiol 233: 5160-5169, 2018.

21. Zendedel A, Monnink F, Hassanzadeh G, Zaminy A, Ansar MM, Habib P, Slowik A, Kipp M and Beyer C: Estrogen attenuates local inflammasome expression and activation after spinal cord injury. Mol Neurobiol 55: 1364-1375, 2018.

22. Chen K, Deng S, Lu H, Zheng Y, Yang G, Kim D, Cao Q and Wu JQ: RNA-seq characterization of spinal cord injury transcriptome in acute/subacute phases: A resource for understanding the pathology at the systems level. PLoS One 8: e72567, 2013.

23. Huang Q, Wu LY, Wang Y and Zhang XS: GOMA: Functional enrichment analysis tool based on GO modules. Chin J Cancer 32: 195-204, 2013

24. Burridge K: Focal adhesions: A personal perspective on a half century of progress. FEBS J 284: 3355-3361, 2017.

25. LaFlamme SE, Mathew-Steiner S, Singh N, Colello-Borges D and Nieves B: Integrin and microtubule crosstalk in the regulation of cellular processes. Cell Mol Life Sci 75: 4177-4185, 2018.
26. De Pascalis C and Etienne-Manneville S: Single and collective cell migration: The mechanics of adhesions. Mol Biol Cell 28: 1833-1846, 2017.

27. Rabelink TJ, van den Berg BM, Garsen M, Wang G, Elkin M and van der Vlag J: Heparanase: Roles in cell survival, extracellular matrix remodelling and the development of kidney disease. Nat Rev Nephrol 13: 201-212, 2017.

28. Bonnans C, Chou J and Werb Z: Remodelling the extracellular matrix in development and disease. Nat Rev Mol Cell Biol 15: 786-801, 2014.

29. Yue B: Biology of the extracellular matrix: An overview. J Glaucoma 23 (8 Suppl 1): S20-S23, 2014.

30. Roy S, Rizvi ZA and Awasthi A: Metabolic checkpoints in differentiation of helper $\mathrm{T}$ cells in tissue inflammation. Front Immunol 9: 3036, 2019.

31. Ridiandries A, Tan JTM and Bursill CA: The role of chemokines in wound healing. Int J Mol Sci 19: pii: E3217, 2018.

32. Wang JG, Williams JC, Davis BK, Jacobson K, Doerschuk CM, Ting JP and Mackman N: Monocytic microparticles activate endothelial cells in an IL-1 $\beta$-dependent manner. Blood 118: 2366-2374, 2011.

33. Prendergast CT and Anderton SM: Immune cell entry to central nervous system-current understanding and prospective therapeutic targets. Endocr Metab Immune Disord Drug Targets 9: 315-327, 2009.

34. Shechter R, London A and Schwartz M: Orchestrated leukocyte recruitment to immune-privileged sites: Absolute barriers versus educational gates. Nat Rev Immunol 13: 206-218, 2013.

35. Demeestere D, Libert C and Vandenbroucke RE: Clinical implications of leukocyte infiltration at the choroid plexus in (neuro)inflammatory disorders. Drug Discov Today 20: 928-941, 2015.

36. Vergadi E, Ieronymaki E, Lyroni K, Vaporidi K and Tsatsanis C: Akt signaling pathway in macrophage activation and M1/M2 polarization. J Immunol 198: 1006-1014, 2017.

37. Kim C, Ye F and Ginsberg MH: Regulation of integrin activation. Annu Rev Cell Dev Biol 27: 321-345, 2011.

38. Pollan SG, Huang F, Sperger JM, Lang JM, Morrissey C, Cress AE, Chu CY, Bhowmick NA, You S, Freeman MR, et al: Regulation of inside-out $\beta 1$-integrin activation by CDCP1. Oncogene 37: 2817-2836, 2018.

39. Dehne N, Fuhrmann D and Brune B: Hypoxia-inducible factor (HIF) in hormone signaling during health and disease. Cardiovasc Hematol Agents Med Chem 11: 125-135, 2013.

40. Risbud MV and Shapiro IM: Notochordal cells in the adult intervertebral disc: New perspective on an old question. Crit Rev Eukaryot Gene Expr 21: 29-41, 2011.

This work is licensed under a Creative Commons Attribution-NonCommercial-NoDerivatives 4.0 International (CC BY-NC-ND 4.0) License. 